\title{
Solar energetic particle interactions with the Venusian atmosphere
}

\author{
Christina Plainaki $^{1, *}$, Pavlos Paschalis ${ }^{2}$, Davide Grassi ${ }^{1}$, Helen Mavromichalaki ${ }^{2}$, and Maria Andriopoulou ${ }^{3}$ \\ ${ }^{1}$ INAF-IAPS, Via del Fosso del Cavaliere, 00133 Rome, Italy \\ ${ }^{2}$ Nuclear and Particle Physics Department, Faculty of Physics, National and Kapodistrian University of Athens, \\ 15784 Athens, Greece \\ ${ }^{3}$ Space Research Science Institute, Austrian Academy of Sciences, Graz, Austria \\ * Invited contribution by C. Plainaki, recipient of the EGU Division Outstanding Young Scientists Award 2014.
}

Correspondence to: Christina Plainaki (christina.plainaki@iaps.inaf.it)

Received: 2 March 2016 - Revised: 20 May 2016 - Accepted: 13 June 2016 - Published: 14 July 2016

\begin{abstract}
In the context of planetary space weather, we estimate the ion production rates in the Venusian atmosphere due to the interactions of solar energetic particles (SEPs) with gas. The assumed concept for our estimations is based on two cases of SEP events, previously observed in near-Earth space: the event in October 1989 and the event in May 2012. For both cases, we assume that the directional properties of the flux and the interplanetary magnetic field configuration would have allowed the SEPs' arrival at Venus and their penetration to the planet's atmosphere. For the event in May 2012, we consider the solar particle properties (integrated flux and rigidity spectrum) obtained by the Neutron Monitor Based Anisotropic GLE Pure Power Law (NMBANGLE PPOLA) model (Plainaki et al., 2010, 2014) applied previously for the Earth case and scaled to the distance of Venus from the Sun. For the simulation of the actual cascade in the Venusian atmosphere initiated by the incoming particle fluxes, we apply the DYASTIMA code, a Monte Carlo (MC) application based on the Geant 4 software (Paschalis et al., 2014). Our predictions are afterwards compared to other estimations derived from previous studies and discussed. Finally, we discuss the differences between the nominal ionization profile due to galactic cosmic-ray-atmosphere interactions and the profile during periods of intense solar activity, and we show the importance of understanding space weather conditions on Venus in the context of future mission preparation and data interpretation.
\end{abstract}

Keywords. Ionosphere (solar radiation and cosmic ray effects)

\section{Introduction}

The atmosphere of Venus is exposed to photon and particle (solar wind, galactic cosmic ray and solar energetic particle (SEP)) radiations, covering a vast range of energies. These populations give rise to a series of interactions between the Venusian atmosphere and the space environment that affect the structure of the atmosphere as well as the gas composition and the energy distribution of its various constituents. While the role of energetic photons has long been recognized (see the recent developments outlined in Gilli et al., 2015), the energetic particles have usually been assumed to have a minor role in the overall atmospheric behaviour. Nonetheless, the interactions between particles (either of galactic or solar origin) and the Venusian atmosphere are expected to have a more crucial role in the modification of the gas properties than that in the case of the Earth or Mars, mainly due to the following reasons:

i. Venus has no significant intrinsic magnetic field; hence, its magnetic cut-off in the rigidity of the incoming charged energetic particles can be considered negligible at all latitudes. As a result, galactic cosmic rays and SEPs are allowed to interact directly with the planet's upper atmosphere and, depending on their energy, to penetrate to deeper layers;

ii. The lower atmosphere on Venus is about 90 times denser (at the surface) than the Earth's; hence, the total amount of the ionized products is expected to be significantly larger than its value in the Earth's case;

iii. Venus is located closer to the Sun; hence, the arriving SEP fluxes during transient solar events (e.g. flares, 
coronal mass ejections - CMEs) are expected to be higher than their values at $1 \mathrm{AU}$, during the same event.

Regarding solar wind, it has been suggested that the field induced by the solar-wind-atmosphere interaction on Venus partially shields the atmosphere at solar maximum (Luhman et al., 2004; Russell et al., 2006). Moreover, Zhang et al. (2007) analysed magnetic field measurements from the Venus Express spacecraft and showed that the bow shock under low solar activity conditions seems to be in the position that would be expected from a complete deflection by a magnetized ionosphere; hence, little solar wind enters the Venus ionosphere even at solar minimum. In view of this, in the current paper, we leave out of our study the investigation of phenomena related to solar wind penetration or deflection by the planet's induced magnetic field.

Due to the relatively denser atmosphere on Venus and to the absence of an intrinsic magnetic field, cosmic ray air showers develop extensively, whereas on Earth many shower products are absorbed by the terrestrial surface. Through such extensive particle cascades, cosmic rays may affect deep atmospheric layers resulting in ion production (at altitudes even lower than $100 \mathrm{~km}$ ) that, furthermore, can potentially affect atmospheric processes such as cloud formation and, in general, atmospheric chemistry (e.g. Dubach et al., 1974; Borucki et al., 1982, Aplin, 2006, 2013; Michael et al., 2009; Nordheim et al., 2015). Intense solar activity, manifested in the presence of solar flares and CMEs, can give rise to an additional component of the particle flux entering the Venusian atmosphere, known also as the solar cosmic ray or SEP component. A flare is a sudden atmospheric brightening on the Sun (see Hudson, 2010, for a review) which produces charged particles and emits increased radiation across the electromagnetic spectrum, particularly in X-ray, extreme ultraviolet (EUV), $\mathrm{H} \alpha$ and radio wavelengths. CMEs are ejections of plasma above the Sun's photosphere, near the corona, commonly associated with solar flares, with speeds typically in the range of $500-1000 \mathrm{~km} \mathrm{~s}^{-1}$. The occasional penetration of solar cosmic rays in the atmosphere of Venus is a significant agent for planetary space weather (Lilensten et al., 2014; Plainaki et al., 2016). In this context, in the current paper, we investigate the Venusian atmosphere interactions with SEPs which, having suprathermal energies, follow trajectories that are influenced only slightly by the planet's induced magnetic field. The full assessment of the role of SEPs in the global behaviour of the Venusian atmosphere is well beyond the scope of an individual paper. As a first step in this process, we focus our attention on the ion pair production rates and the accurate estimation of the characteristics of the atmospheric cascades initiated by SEPs bombarding the Venusian atmosphere. The main motivation of the current work, therefore, is to provide a body of information related to SEP-atmosphere interactions on Venus (e.g. the ionization rate profile) during different solar activity conditions that can be used as inputs in future sophisticated studies related, for example, to electrical con- ductivity or the charging of cloud particles. Finally, the study of the particle-atmosphere interactions on Venus may reveal processes fundamental to the interpretation of exoplanet observations. As many exoplanets are located only a few stellar radii away from their parent stars, the effects and type of interactions under such conditions are among the key science questions to be answered in the near future.

Some important efforts to study the cosmic ray interactions with the Venusian atmosphere have already been made in the past. Dubach et al. (1974), Borucki et al. (1982), and Upadhyay and Singh (1995) have made use of approximate transport equations to describe particle propagation within the Venusian atmosphere. Whereas these works provided an important starting point, Nordheim et al. (2015) noted that simplifications that generally do not take into account the full range of effects due to the interactions of primary and secondary air shower components with atmospheric neutrals were implied. Nordheim et al. (2015) used the PLANETOCOSMICS software application (Desorgher et al., 2005) to perform a Monte Carlo modelling of cosmic ray interactions within the Venusian atmosphere, including discrete particle interactions within the extensive showers of secondary particles and taking into account the contribution from protons, alpha particles and heavier ions. In the same context, in the current study we propose the use of a new Monte Carlo (MC) application in order to calculate the atmosphere ionization (and ion pair production) rate profile due to SEPs and to identify its dependence on the energy spectrum of the impinging particles. The Dynamic Atmospheric Shower Tracking Interactive Model Application (DYASTIMA) used in the current work is based on the Geant4 toolkit, and it has been previously applied for the Earth case for both galactic cosmic rays (Paschalis et al., 2014) and SEP (Dorman et al., 2015) interactions with the terrestrial atmosphere. In the context of planetary space weather, the use of DYASTIMA in the estimations in the current paper allows us, for the first time, to consider SEP-related information derived from global ground-based measurements obtained on Earth (see Sect. 2.2. for details) and scaled to Venus' distance from the Sun (i.e. 0.72 AU). Moreover, in the current work we attempt to distinguish, for the first time, the contribution of the high-energy incoming particles, during an SEP event, to the atmosphere ionization rate profile from the contribution of the lower-energy population. To reach this goal we use a realistic SEP spectrum already tested for the Earth case, based on data corresponding to a vast SEP energy range (see Sect. 2.2), assuming a priori that the directional properties of the solar particles allow their arrival and penetration to the Venusian atmosphere. We examine firstly the case of the energetic solar event in October 1989, and our results are compared to those obtained by Nordheim et al. (2015) for the same event. Additionally to the implementations in Nordheim et al. (2015), we study also the case of the recent SEP event on 17 May 2012. Given its characteristics (see, for example, Li et al., 2013, and Gopalswamy et al., 2013), the proton event on 17 May 2012 
can be considered a middle case between a very energetic (up to several $\mathrm{GeVs}$ ) and a less energetic (in the range of a few tens of MeVs up to some hundreds of MeVs) SEP event. In this context, the results provided here are an important addon to current knowledge and can be further used in planetary space weather studies related to terrestrial planets possessing atmospheres. To study the effects in the Venusian atmosphere of an SEP event of the order of magnitude (in both energy and flux) of the event on 17 May 2012 we consider the solar particle properties (integrated flux and spectrum) obtained by the Neutron Monitor Based Anisotropic GLE Pure Power Law (NMBANGLE PPOLA) model (Plainaki et al., 2010, 2014) applied previously for the Earth case and scaled to the distance of Venus. Moreover, in order to investigate the differences between the nominal ionization profile due to galactic cosmic-ray-atmosphere interactions and the one during SEP events, we perform two additional simulations of the expected cascades during solar minimum and solar maximum conditions. This paper is organized as follows: in Sect. 2 we provide a brief description of both basic models used in this study, namely DYASTIMA and NMBANGLE PPOLA, and we present the input parameters considered in the simulations. In Sect. 3 we present and discuss our results corresponding to both SEP event cases, and we compare (where possible) our predictions to those obtained in previous works. In Sect. 4 we discuss the usefulness of these estimations in further investigations of an interdisciplinary nature, related also to future space-based observations. Finally, in Sect. 5 we present the main conclusions of the current work.

\section{Brief description of the models used}

\subsection{DYASTIMA and inputs}

DYASTIMA is an application, based on Geant4, which simulates the cascades of particles generated after the interactions of galactic cosmic ray particles and/or SEPs with the atmosphere of a planet. The DYASTIMA code has been implemented at the Athens Neutron Monitor Station (ANEMOS) of the National and Kapodistrian University of Athens (Paschalis et al., 2014), and the corresponding application is available online. ${ }^{1}$ The first version of DYASTIMA has been successfully applied to the Earth's atmosphere, providing results that are in accordance with the publications of other models (Paschalis et al., 2014). DYASTIMA allows for (1) the possibility of consideration of complex scenarios, as inputs, including for example user-defined vertical profiles of atmospheric quantities such as densities and mixing ratios; (2) the possibility to define the primary energy spectra of the impinging cosmic ray particles; (3) the obtaining of enhanced outputs, such as the density profiles of secondary products of atmospheric cascades. In this section

\footnotetext{
${ }^{1}$ DYASTIMA can be downloaded from http://cosray.phys.uoa. gr/index.php/applications/dyastima.
}

we briefly discuss the main characteristics of DYASTIMA, and we describe the considered inputs during its application to the Venusian atmosphere. For more details on the DYASTIMA application and its techniques, the reader may refer to Paschalis et al. (2014).

The study of particle cascades via simulations is a complicated procedure due to the numerous input and output parameters that need to be considered. Up to now various techniques have been implemented (e.g. the use of approximate transport equations to describe particle propagation within the atmosphere; MC techniques simulating a vast number of individual events). For the Earth's case there is a vast body of literature on atmospheric cascade models: very often the FLUKA (Battistoni et al., 2007) or the Geant 4 (Agostinelli et al., 2003; Allison et al., 2006) simulation toolkits are used, but the CRII model calculating the cosmicray-induced ionization in the atmosphere is very important as well (Usoskin et al., 2004, 2010). CORSIKA (Heck et al., 1998) is a MC program used for the detailed study of extended atmospheric shower evolution and of the showers' properties. The Geant4-based ATMOCOSMICS code (Desorgher et al., 2005), usually combined with MAGNETOCOSMICS, simulates the interaction of cosmic rays with the Earth's atmosphere, allowing the computation of the flux of secondaries at different atmospheric depths and/or altitudes. PLANETOCOSMICS is a more evolved code with respect to MAGNETOCOSMICS and ATMOCOSMICS, extensively applied in the field of planetary cosmic ray studies, including Mars (Gronoff et al., 2015; Ehresmann et al., 2011; Gurtner et al., 2005), Mercury (Gurtner et al., 2006), Venus (Nordheim et al, 2015; Dartnell et al., 2015) and Titan (Gronoff et al. (2009a, b, 2011). The recently developed DYASTIMA code includes many of the same features considered in PLANETOCOSMICS (e.g input of a custom atmospheric description, detection of primary and secondary fluxes and directions at given altitudes, user-defined primary cosmic ray spectra). Indeed, within DYASTIMA a series of phenomena taking place in a planetary atmosphere during and after its interactions with the incoming charged particle fluxes can be parameterized; hence, different simulation scenarios (considering, for example, the primary cosmic ray spectrum, the atmospheric structure and the magnetic field) can be easily considered. On the other hand, the output of DYASTIMA provides all the available information about the number, the energy, the direction and the arrival time of the secondary particles. Overall, DYASTIMA is an easy to use application providing several pieces of output information that can be fitted to a variety of external applications. The most indicative input parameters that should be managed, combined and transformed in order to be inserted in the application, are the structure of the atmosphere (density, mixing ratios of different components), the magnetic field, the spectra of the incoming particles, the physical interactions that are considered, the energy channels that are predefined in order to accelerate the simulation, and the altitudes in which the in- 
Table 1. Input parameter values considered in DYASTIMA during its application to the Venusian atmosphere, for solar minimum and solar maximum conditions.

\begin{tabular}{|c|c|c|}
\hline \multicolumn{3}{|c|}{ Planetary properties } \\
\hline Parameter & Value & Comments \\
\hline Planet radius & $6052 \mathrm{~km}$ & $\begin{array}{l}\text { NASA Venus fact sheet } \\
\text { http://nssdc.gsfc.nasa.gov/planetary/factsheet/venusfact.html }\end{array}$ \\
\hline World volume width & $500 \mathrm{~km}$ & \\
\hline Geometry model & SPHERE & \\
\hline Air density change & $10 \%$ & \\
\hline Physics list & FTFP_BERT_HP & $\begin{array}{l}\text { It uses Bertini-style Cascade }(\sim<10 \mathrm{GeV}) \text {, high-precision neu- } \\
\text { tron model }(<20 \mathrm{MeV}) \text { and Fritiof model for high energies } \\
(>4 \mathrm{GeV}) \text {. All standard electromagnetic processes are included. }\end{array}$ \\
\hline Range cut & $1 \mathrm{~m}$ & \\
\hline Surface pressure & $92100 \mathrm{mb}$ & \\
\hline Gravity acceleration at the surface & $8.869 \mathrm{~m} \mathrm{~s}^{-2}$ & $\begin{array}{l}\text { NASA Venus fact sheet } \\
\text { http://nssdc.gsfc.nasa.gov/planetary/factsheet/venusfact.html }\end{array}$ \\
\hline Spectrum altitude & $150 \mathrm{~km}$ & \\
\hline $\begin{array}{l}\text { Atmosphere Composition } \\
\text { Parameter }\end{array}$ & Value & Comments \\
\hline Section number & 1 & \\
\hline Section start/end & $0 / 150 \mathrm{~km}$ & \\
\hline Molecule/vol abundance & $\mathrm{CO}_{2} / 96.5 \% \mathrm{~N}_{2} / 3.5 \%$ & Kliore et al. (1985); Seiff et al. (1985); Keating et al. (1985) \\
\hline
\end{tabular}

formation about the cascade will be registered. The physics list used in DYASTIMA contains all standard electromagnetic processes (see Table 1).

In the current work, the assumed atmospheric composition on Venus is $96.5 \%$ vol $\mathrm{CO}_{2}$ and $3.5 \%$ vol $\mathrm{N}_{2}$. The atmospheric description is based on the Venus International Reference Atmosphere (Kliore et al., 1985) and on the estimations by Seiff et al. (1985) for the middle and lower atmosphere $(100-0 \mathrm{~km})$ at low latitudes $\left(u<30^{\circ}\right)$ and on those by Keating et al. (1985) for the daytime upper atmosphere between $100-150 \mathrm{~km}$ at low latitude $\left(u=16^{\circ}\right)$. We note that the considered atmospheric composition and densities are the same as in Nordheim et al. (2015). The energy spectrum of the incoming galactic cosmic rays was obtained with CREME2009 (https://creme.isde.vanderbilt.edu/) (Tylka et al., 1997), in the range of $\sim 1 \mathrm{MeV}$ up to $100 \mathrm{GeV}$ in interplanetary space at $1 \mathrm{AU}$. The galactic cosmic ray component of the CREME2009 model is based on the International Standard Galactic Cosmic Ray Model of the International Organization for Standardization (ISO 15390:2004(E)) (Nymmik, 2006), with additional extensions, including the treatment of anomalous cosmic rays at low energies (Tylka et al., 1997). In this work we considered the galactic cosmic ray spectrum for "solar quiet" conditions at solar maximum and minimum, which represent ambient conditions in the absence of SEP events (Nordheim et al., 2015). As the gradient of galactic cosmic ray flux is very low within the inner solar system (Fujii and McDonald, 1997), rescaling of the CREME2009 galactic cosmic ray fluxes to the orbit of Venus has not been considered in this study. We note that the galactic cosmic ray energy spectrum considered here is the same as the one considered in Nordheim et al. (2015), including also alpha particles and heavier ions. All primary and secondary particles are tracked until they either come to rest within the atmospheric column or are absorbed by the planetary surface. The settings used in DYASTIMA during its application to the Venusian atmosphere are summarized in Table 2.

\subsection{NMBANGLE PPOLA and inputs for the SEP event cases under study}

The NMBANGLE PPOLA model (Plainaki et al., 2010, 2014,2015 ) is a modified version of the original NMBANGLE model (Plainaki et al., 2007), based on the coupling coefficient method (Dorman, 2004). Both models have been applied numerous times in the past (e.g. Plainaki et al., 2007, $2009,2010,2014$ ) with the scope to obtain the SEP properties during a solar event at the distance of $1 \mathrm{AU}$ from the Sun. The NMBANGLE PPOLA model couples SEPs (primary particles) at a selected altitude in the Earth's atmosphere with the secondary particles detected by ground-based neutron monitors during SEP events. This model dynamically calculates the SEP spectrum and the SEP flux spatial distribution at a particular altitude in the atmosphere through the simulation of the response of the terrestrial atmosphere to this primary population. A power-law SEP spectrum with two free parameters (spectral index and amplitude) is assumed. SEPs are assumed to be protons in the model's current version. The 
Table 2. Input parameter values used in DYASTIMA during its application to the October 1989 and May 2012 SEP events.

\begin{tabular}{|c|c|c|}
\hline Parameter & October 1989 event & May 2012 event \\
\hline Simulated time periods & $\begin{array}{l}\text { 19-26 October } 1989 \text { (in total } 180 \mathrm{~h} \text {, begin- } \\
\text { ning } \\
\text { at 13:00 UT): "worst week"c }\end{array}$ & $\begin{array}{l}17 \text { May } 201202: 00-02: 05 \mathrm{UT} \text { (peak phase for the } \\
\text { relativistic proton energies }>\sim 0.5 \mathrm{GeV} \text { ) } \\
17 \text { May } 201203: 25-03: 30 \mathrm{UT}(\text { arbitrary selected time lag } \\
\text { belonging to the event's "main phase" for } \\
\text { energies }>100 \mathrm{MeV})^{\mathrm{c}}\end{array}$ \\
\hline $\begin{array}{l}\text { Energy range of } \\
\text { impinging solar protons }\end{array}$ & $10-800 \mathrm{MeV}^{\mathrm{c}}$ & $100 \mathrm{MeV}-1.7 \mathrm{GeV}^{\mathrm{d}}$ \\
\hline \multicolumn{3}{|c|}{$\begin{array}{l}\text { Based on the available satellite observations. } \\
\text { Based on estimations using the NMBANGLE PPOLA space weather model. The NMBANGLE PPOLA results for the event's spectrum (Plainaki et al., 2014) were obtained at } \\
\text { elativistic proton energies }>\sim 0.5 \mathrm{GeV} \text {. At lower energies the data were obtained from extrapolation of the model results. The SEP energy spectrum was scaled to Venus' distance } \\
\text { rom the Sun. See text for details. } \\
\text { Selection based on the time series of the available GOES observations (Verronen et al., 2002) elaborated within the model (Tylka et al., 1997). See text for details. } \\
\text { An approximate upper limit for the energy of the solar protons released during the event in May } 2012 \text { was obtained from the analysis of ground-based neutron monitor } \\
\text { neasurements of the worldwide network (see, for details, Plainaki et al., 2014) in consistency also with estimations obtained in other studies (e.g. Li et al., 2013). The same upper } \\
\text { energy for the SEPs was assumed in the current simulations. }\end{array}$} \\
\hline
\end{tabular}

details of the physics considered in the NMBANGLE and the NMBANGLE PPOLA models have been provided in the past (see Plainaki et al., 2007, 2014). In this section we only give a very brief description of the model and discuss the necessary adaptation of its results in order to investigate the response of the Venusian atmosphere to a specific event.

The NMBANGLE PPOLA model, when applied for the Earth case, uses as inputs the response of the worldwide neutron monitor network to the high-energy solar protons (i.e. $\geq \sim 500 \mathrm{MeV}$ ) and the disturbance level of the geomagnetic field (through the use of the $\mathrm{Kp}$ index). A neutron monitor is a ground-based instrument that records cosmic rays originating either from the galaxy or from the Sun and travelling through the interstellar or interplanetary space. In particular, a neutron monitor detects secondary particles generated after the collisions of primary impinging particles (of galactic or solar origin) with the nuclei of atmospheric atoms. Because the intensity of cosmic rays hitting the Earth's atmosphere is in general not uniform, it is important to have neutron monitors placed at different locations in order to obtain a complete picture of the spatial and energy distribution of the primary populations. In this context, the NMBANGLE PPOLA model treats the existing neutron monitor network (for a detailed description, see Mavromichalaki et al. (2011) and Plainaki et al. (2014) and references therein) as an integrated omnidirectional spectrometer able to measure the characteristics of the relativistic primary flux at a selected altitude in the Earth's atmosphere. In particular, in the NMBANGLE PPOLA model, for the time period of an SEP event, groundbased data from neutron monitor stations widely distributed around the world are incorporated. The incoming charged particle motion inside the geomagnetic field is taken into account and used as an input parameter in the model. Through the solving of the inverse problem, the model calculates the spectral index and the amplitude of the incoming SEP flux at some altitude in the Earth's atmosphere, the direction of the maximum flux and the spread of the actual flux around this location (for example, the model can reveal whether an event is highly anisotropic, i.e. with a small spread around the location of the maximum SEP flux, or less anisotropic (i.e. with a wide spread)). The reader is requested to refer to Plainaki et al. (2007) and Plainaki et al. (2015) for a detailed description of how the free parameters of the model connect to the actual physical characteristics of the SEP event.

In the current paper, we investigate the response of the Venusian atmosphere to two different cases of SEP events (i.e. in October 1989 and in May 2012), assuming that the directional properties of those solar particles allow their arrival and penetration to the gas. Whereas for the recent event of May 2012 we make use of SEP information derived directly from the NMBANGLE PPOLA (see below for details), for the event in October 1989 we use the same SEP-related information as in Nordheim et al. (2015), obtained with CREME2009. Considering the SEP event in October 1989, the use of the same inputs as the ones in the work by Nordheim et al. (2015), allows the possibility of a direct validation of our code, since both works refer to the same space weather event. Considering the May 2012 event, we choose to make use of the NMBANGLE PPOLA results because they are derived using data from an extended neutron monitor network (Mavromichalaki et al., 2011); hence, allowing the direct reconstruction of the SEP energy spectrum in a proton energy range extending up to a few GeVs (Plainaki et al., 2014). The October 1989 event is known as one of the most intense solar events ever observed (Miroshnichenko et al., 2000). The SEP spectrum assumed in this work cor- 
responds to the "worst week" scenario, as in Nordheim et al. (2015). Such a scenario corresponds to particle fluxes averaged over $180 \mathrm{~h}$ beginning at 13:00 UTC on the 19 October 1989 (Tylka et al., 1996). The SEP fluxes obtained with CREME2009 are based on the use of solar particle models all based on GOES proton data and high-energy heavy-ion measurements from the University of Chicago's Cosmic Ray Telescope (CRT) on the Interplanetary Monitoring Platform 8 (IMP-8). Proton and heavy-ion measurements for this event extended to $\sim 500-800 \mathrm{MeV}$, depending on species (Tylka et al., 1997). Although there was evidence that higher energies than $800 \mathrm{MeV}$ did indeed exist during that event (Cramp et al., 1997), the CREME2009-based SEP spectrum we use in the current study forces us to set the upper limit for the impinging particle energy to $\sim 800 \mathrm{MeV}$. Such a choice of course influences physically the results for the atmosphere ionization (see the "Results and discussion" section); nevertheless, given the high uncertainty in the 1989 satellite measurements, the use of the current SEP spectrum can be considered as a best-effort scenario.

Regarding the event on 17 May 2012, we examine the ionization provoked by two distinct SEP populations, those of high energy, i.e. $>\sim 0.5 \mathrm{GeV}$, and those at energies in the range from a few $\mathrm{MeV}$ up to about $0.5 \mathrm{GeV}$. For the first population, we consider the results obtained from the NMBANGLE PPOLA application and, in particular, the SEP energy spectrum outside the terrestrial atmosphere, scaled to Venus' distance from the Sun using a geometrical $\left(1 / R^{2}\right)$ factor. In particular, the SEP energy spectrum considered as input for the DYASTIMA application is obtained from the average over all latitude and longitude spectra obtained from the NMBANGLE PPOLA model application for (1) the peak phase (at high energies) of the event and (2) the main phase of the event (see Fig. 3 in Plainaki et al., 2014). The consideration of differential flux averaged for angles is a reasonable choice since any spatial anisotropy in the SEP flux registered at $1 \mathrm{AU}$ does not necessarily occur at $0.72 \mathrm{AU}$. The second SEP population is actually the one registered by satellites (e.g. GOES and ACE); nevertheless, the low-energy resolution of the low-energy flux measurements during the event of 17 May 2012 does not allow their use in DYASTIMA. For this reason, in the low-energy range, we choose to use the extrapolated SEP spectrum obtained initially with the NMBANGLE PPOLA model. As previously, here the necessary scaling factor, due to Venus' distance from the Sun, is also applied to the SEP fluxes. We underline that such a choice may lead to an overestimation of the actual low-energy flux (see Fig. 6 and discussion in Plainaki et al., 2014); nevertheless, comparison of the modelled low-energy fluxes with the ones obtained from the satellites at the event's peak phase are in relatively good agreement, for the Earth case (Plainaki et al., 2015). Since for both SEP populations the aim of the simulation corresponding to the May 2012 solar event is to examine the properties of the atmospheric cascade during a moderate SEP event on Venus, we consider that the use of the scaled NMBANGLE PPOLA spectrum as an input does not lead to significant errors in the overall estimation. Analyses including detailed modelling of the particle propagation in the interplanetary space, incorporating sophisticated modes (e.g. ENLIL, Odstrcil, 2003), go beyond the scope of the current paper. The inputs for the DYASTIMA application considering SEP fluxes are summarized in Table 2.

\section{Results and discussion}

The simulations performed with DYASTIMA considering the inputs presented in Tables 1 and 2 allow the computation of the total energy deposition versus altitude in the Venusian atmosphere. On the basis of this information, the atmospheric density and the mean ionization potential (assumed here to be equal to $33.5 \mathrm{eV}$, i.e. equal to the energy needed for the creation of one ion-electron pair in $\mathrm{CO}_{2}$ according to Borucki et al., 1982), we calculate the ionization rate as a function of altitude.

\subsection{Ionization due to SEP-atmosphere interactions}

Assuming that the directional properties of the solar particles during the event on 17 May 2012 would have allowed their arrival and penetration to the Venusian atmosphere, we present in Fig. 1 the expected ionization profiles. Since the maximum energy or rigidity of the SEPs arriving at the Earth's vicinity on 17 May 2012 is likely equal to $1.7 \mathrm{GeV} / 2 \mathrm{GV}$ (Plainaki et al., 2014), the input spectrum considered in the current simulations extends the energy range from $100 \mathrm{MeV}$ (approximate overall lower limit for the NMBANGLE PPOLA model) to $1.7 \mathrm{GeV}$. The estimations in Fig. 1 have been provided for the conditions at the "peak phase" of the $5 \mathrm{~min}$ averaged fluxes at proton energies greater than $\sim 500 \mathrm{MeV}$, as observed at $1 \mathrm{AU}$ and/or modelled. ${ }^{2}$ In Fig. 1, we have distinguished the contribution due to higherenergy-charged particles from the one due to lower-energy ones (a value equal to $\sim 500 \mathrm{MeV}$ was considered as the border between these two populations, consistent also with the energy range in which the cosmic ray spectrum is subject to solar modulation). As seen in Fig. 1, at higher altitudes $(>71 \mathrm{~km})$ in the Venusian atmosphere the contribution to the ionization due to lower-energy SEPs dominates. At lower altitudes, the more energetic particles have a major role in ionization, resulting in a rate of $\sim 70$ ion pairs $\mathrm{cm}^{-3} \mathrm{~s}^{-1}$ at the altitude of $67 \mathrm{~km}$. The total maximum of ionization due to both solar particle populations is $\sim 85$ ion pairs $\mathrm{cm}^{-3} \mathrm{~s}^{-1}$ and is expected at the altitude of $70 \mathrm{~km}$. During later phases of the event, when the spectrum was softer (Plainaki et al., 2014; Mishev et al., 2014), the ionization profile is more intense

\footnotetext{
${ }^{2}$ The identification of the "peak phase" has been done through the modelling of the high-energy part of the SEP spectrum using ground-based neutron monitor measurements from the worldwide network of neutron monitors (see, for details, Plainaki et al., 2014).
} 


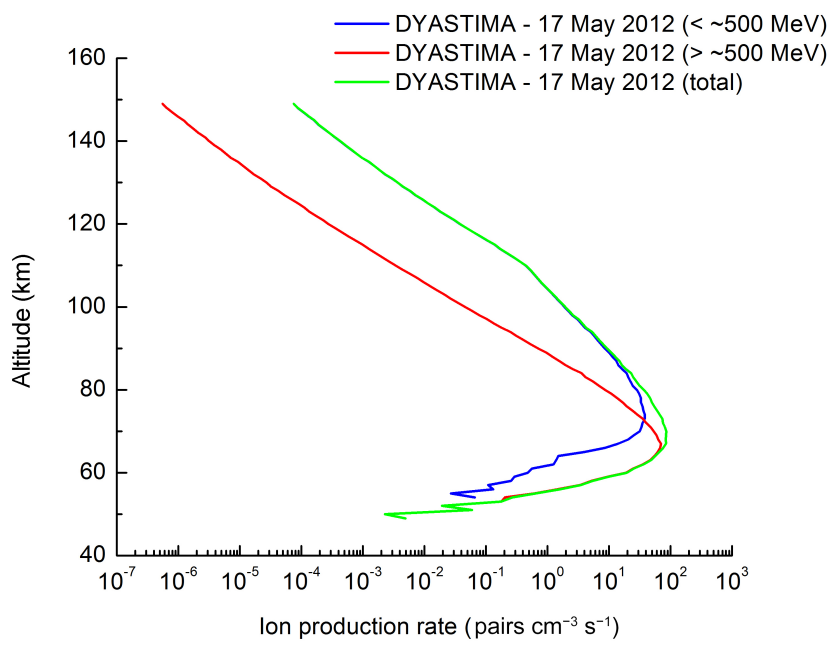

Figure 1. Ionization rate profiles in the Venusian atmosphere due to the arrival and penetration of SEPs during the event on 17 May 2012. The results obtained from the NMBANGLE PPOLA model, scaled to the distance of Venus, were considered as inputs in the DYASTIMA application. The estimations presented here correspond to the "peak phase" of the event, considered as the time that the $5 \mathrm{~min}$ averaged fluxes at proton energies greater than $500 \mathrm{MeV}$ (as observed at $1 \mathrm{AU}$ and/or modelled) reached their maximum flux.

and the maximum ionization takes place at higher altitudes. In particular, in Fig. 2, the results of the application of DYASTIMA to the May 2012 SEP event refer to the main phase of the event, considered here as the time of the peak of the fluxes at energies of at least $100 \mathrm{MeV}$. The results show that the maximum ionization rate appears at the altitude of $78 \mathrm{~km}$ and is equal to $\sim 396$ ion pairs $\mathrm{cm}^{-3} \mathrm{~s}^{-1}$. Based on these results it becomes clear that variabilities in the SEP fluxes during a solar event can provoke significant modifications in the ionization efficiencies in the Venusian atmosphere. Based on these results, it becomes clear that it is important to have knowledge of the actual planetary space weather conditions around Venus when interpreting atmosphere measurements.

In addition to the DYASTIMA results corresponding to the May 2012 event's main phase, in Fig. 2 we also present the ionization profiles in the Venusian atmosphere due to the arrival and penetration of SEPs during the event in October 1989 (worst week). For comparison we include also the estimations by Nordheim et al. (2015) referring to the October 1989 event (worst week). It is clearly seen that the results obtained with DYASTIMA and the ones by Nordheim et al. (2015) are very similar. In particular, using the DYASTIMA code, we find that the maximum ionization rate appearing at the altitude of $94 \mathrm{~km}$ is equal to 7164 ion pairs $\mathrm{cm}^{-3} \mathrm{~s}^{-1}$. Nordheim et al. (2015) found the ionization peak at $95 \mathrm{~km}$, equal to $\sim 7000$ ion pairs $\mathrm{cm}^{-3} \mathrm{~s}^{-1}$.

During the event in May 2012, lower ionization rates are expected (by a factor of $\sim 18$ at the ionization maximum) with respect to their values during the event in 1989. This is

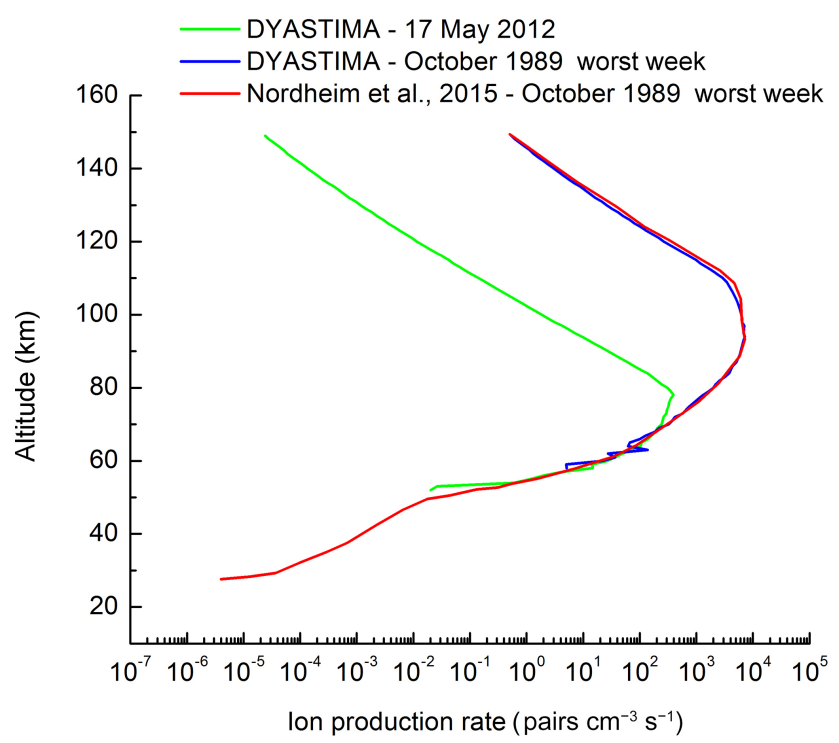

Figure 2. Ionization rate profiles in the Venusian atmosphere due to the arrival and penetration of SEPs during different events. For the event in October 1989 the results of the current study obtained with DYASTIMA (blue line) and the results by Nordheim et al. (2015) (red line), both refer to the event's "worst week". The results of the application of DYASTIMA to the May 2012 SEP event (green line) refer to the "main phase" of the event, i.e. after the peak time of the low-energy (of at least $100 \mathrm{MeV}$ ) proton fluxes (see also Table 2).

due to the relatively less intense SEP fluxes occurring during the 2012 event. Nevertheless, the maximum of the ionization rate is at a lower altitude during the event of 2012 than during the 1989 event. Such a difference is justified on the basis of the SEP spectrum used as an input in the DYASTIMA simulations, extended to higher energies (up to $1.7 \mathrm{GeV}$ ) when applied to the 2012 event than when applied to the 1989 event (up to $800 \mathrm{MeV}$ ). As mentioned also in Sect. 2.2, our choice for the input SEP spectrum for the 1989 event was limited due to the outputs provided by the CREME2009 model. However, there is evidence that the actual SEP energies extended to $\geq 1 \mathrm{GeV}$ (Cramp et al., 1997). Therefore, the DYASTIMA results corresponding to the 1989 event, as well as those by Nordheim et al. (2015), are only approximate. We predict that in the case of a more realistic SEP spectrum (i.e. extending to higher proton energies) being used, the ionization profile corresponding to the event in 1989 would have a maximum at a somewhat lower altitude. By contrast, we would not expect a significant variation in the ionization rate intensity. This is because relevant studies performed for the Earth case have shown that within the range of SEP energies (and in particular in the typical range $200 \mathrm{MeV}-1 \mathrm{GeV}$ ), the actual cross section of the proton-air interaction increases rapidly with decreasing energy (see Fig. 4.2 in Heck et al., 1998; Fesefeldt, 1985). 


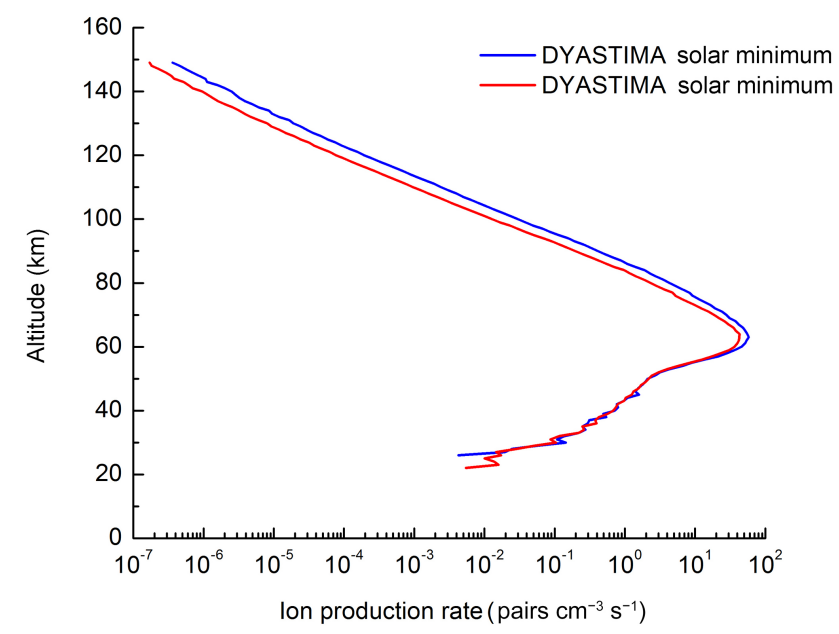

Figure 3. Ionization rate profiles in the Venusian atmosphere, as a function of altitude, derived with the DYASTIMA application. The interactions of the galactic cosmic rays with the gas were examined for both solar minimum and solar maximum conditions.

\subsection{The ionization background induced by galactic cosmic rays}

In Fig. 3 we present the ionization profile in the Venusian atmosphere due to galactic cosmic rays during solar minimum and solar maximum conditions, as derived from the application of DYASTIMA. Our results show a peak equal to $\sim 58$ ion pairs $\mathrm{cm}^{-3} \mathrm{~s}^{-1}$ at $63 \mathrm{~km}$ at solar minimum and a peak equal to $\sim 43$ ion pairs $\mathrm{cm}^{-3} \mathrm{~s}^{-1}$ at $64 \mathrm{~km}$ at solar maximum. We note that the profiles above these altitudes are very steep, whereas, at altitudes lower than $\sim 56 \mathrm{~km}$, the ionization rates for both cases are quite similar. This result was indeed expected since only the higher-energy cosmic ray particles, which are not subject to solar modulation, can penetrate to deep atmospheric layers. At solar minimum, above the altitude of $\sim 56 \mathrm{~km}$, the ionization profiles are consistently higher than those at solar maximum by a factor higher or equal to 1.3. This is a natural consequence of the harder galactic cosmic ray spectrum during solar maximum.

In Fig. 4 we present the ionization profile in the Venusian atmosphere due to galactic cosmic rays, as derived from the application of the DYASTIMA code, in comparison with the estimations in other studies. We note that at most altitudes our results are practically identical to those by Nordheim et al. (2015), which is due to the similar inputs (e.g. atmospheric composition and densities) and codes (based on Geant4) used in both studies. We note that at low atmospheric altitudes $(<\sim 45 \mathrm{~km})$, where statistics are poor compared to those at lower atmospheric depths, the variations in the rates estimated with DYASTIMA are likely due to statistical noise inherent in the Monte Carlo approach. The work by Borucki et al. (1982) predicted an ionization peak that is $\sim 28 \%$ higher in magnitude than the one in the present study, occurring nevertheless at almost the same altitude, i.e. at $64 \mathrm{~km}$. In

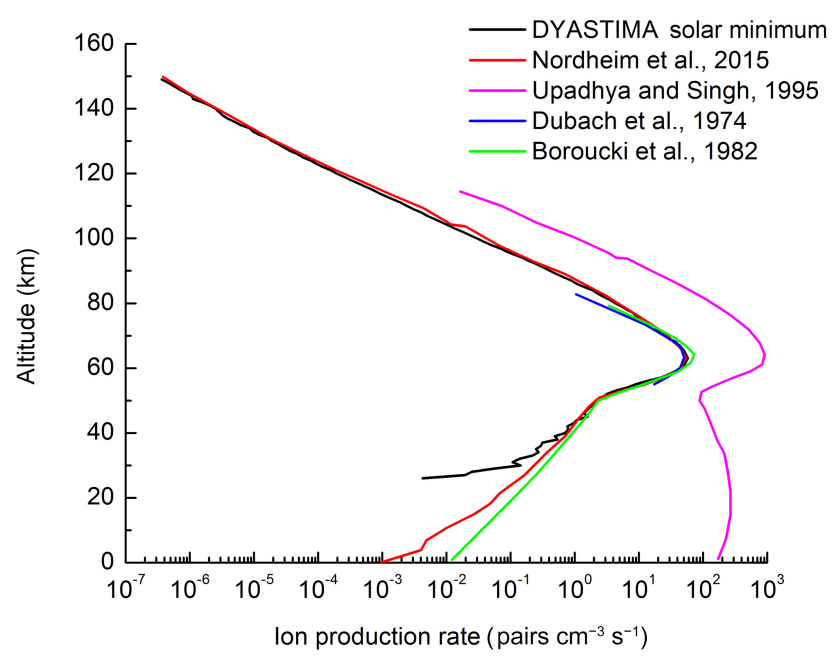

Figure 4. Ionization rate profiles in the Venusian atmosphere, as a function of altitude, derived with the DYASTIMA application for solar minimum conditions, in comparison with the estimates in other studies.

the work by Dubach et al. (1974) a peak ionization rate equal to $\sim 49$ ion pairs $\mathrm{cm}^{-3} \mathrm{~s}^{-1}$ was estimated. This value is lower than the estimate in the current study, but it is worth noticing that Dubach et al. (1974) also find this peak at the same altitude as the one our model predicts, i.e. at $63 \mathrm{~km}$. Both studies by Borucki et al. (1982) and Dubach et al. (1974) made use of approximate solutions to the Boltzman transport equation. Since such a method is less reliable at large atmospheric depths (Nordheim et al., 2015), their ionization rates at low atmospheric altitudes are higher than our predictions as well as those by Nordheim et al. Above $\sim 70 \mathrm{~km}$ the ionization rates by Borucki et al. (1982) and Dubach et al. (1974) are lower than the values in this study and in the study by Nordheim et al. (2015). For comparison we include also the results by Upadhyay and Singh (1995), who found a significantly higher ionization peak with respect to the ones estimated in the other studies, equal to $10^{3}$ ion pairs $\mathrm{cm}^{-3} \mathrm{~s}^{-1}$ at the altitude of $64 \mathrm{~km}$ and a broad muon-related ionization peak in the lower-altitude atmosphere. No previous or subsequent study shows the same sort of ionization profile as predicted by that paper. Indeed, this is also the case for the results presented in this manuscript, which are broadly consistent with the other previous ones. Maybe these discrepancies are due to different values of the average ionization energy (equal to $\sim 11.5 \mathrm{eV}$ in Upadhyay and Singh (1995) and equal to $\sim 33.5 \mathrm{eV}$ in the current study). As Nordheim et al. (2015) noted, however, a complete and in depth comparison among models is not possible since the coverage in altitudes is not the same among all these studies.

The ion pair production rates by galactic cosmic rays provided in this paper show peaks ranging between 63 and $64 \mathrm{~km}$ depending on the solar activity cycle. A number of phenomena can be potentially related to this production. 
The presence of intense ionization at lower altitudes with respect to the ones corresponding to the Earth's case suggest the possibility of lightning discharge in Venus' atmosphere. Indeed, several studies suggest lightning discharges above, between or within the clouds of the Venusian atmosphere (Borucki, 1982; Gurnett et al., 2001). The reports of observations indicating Venusian lightning (for example through radio wave detection) are limited (see Ksanfomaliti et al., 1979; Russell et al., 2007; Taylor et al., 1979a, b) and the details of the exact mechanism causing lightning in the atmosphere are largely unknown. Cloud-to-ground discharges are unlikely in the warmer and denser regions of the atmosphere of Venus (Aplin, 2006; Gurnett et al., 2001).

The large datasets collected by Venus Express instruments have recently stimulated important efforts on theoretical modelling of aerosol formation and growth in the Venusian atmosphere and comparison with experiments (see recent examples in Merikanto et al., 2016; Duplissy et al., 2016; and Määttänen et al., 2015). In this context, the simulation results in this study can be used as complementary inputs, since we confirm here that cosmic rays can indeed ionize the Venusian atmosphere at large depths, as demonstrated in previous studies (e.g. Dubach et al., 1974; Borucki et al., 1982; Aplin, 2006, 2013; Michael et al., 2009; Nordheim et al., 2015). Such ionization processes appear well within the region of aerosol occurrence, possibly enhancing the efficiency of condensation nuclei.

Several datasets from Venus Express suggest the occurrence of relatively rapid variations (a few days) in the air temperature in the lower thermosphere (indicatively 100$120 \mathrm{~km}$ ). These phenomena have been reported in the analysis of the SPICAV/SOIR (Spectroscopy for Investigation of Characteristics of the Atmosphere of Venus/Solar Occultation in the Infrared) (Piccialli et al., 2015; Mahieux et al., 2015) and VIRTIS (Visible and Infrared Thermal Imaging Spectrometer) (Peralta et al., 2016) data. Notably, Peralta et al. (2016) did not find any correlation between rapid temperature variations and overall solar activity as measured by solar radio flux at $10.7 \mathrm{~cm}$ (considered as a proxy for the overall UV flux). Our results and those of Nordheim (2015) in Fig. 4 suggest, however, that these altitudes can be prone to important ionization at least in the case of major SEP events. Future investigation on the possible role of SEPs in the observed rapid variations in the air temperature in the lower Venusian thermosphere may provide some feedback in this direction.

The maximum ionization due to galactic cosmic rays occurs at lower altitudes in the Venusian atmosphere (see Fig. 3 and Sect. 3.1) than the one due to SEP events. This happens because the energy-dependent cross section of the proton-air interaction increases rapidly with decreasing energy in the $0.2-1 \mathrm{GeV}$ range, whereas it remains consistently lower in the energy range from $2 \mathrm{GeV}$ up to $100 \mathrm{GeV}$ (see Fig. 4.2. in Heck et al., 1998). As a result, the more energetic an impinging charged particle is, the less it interacts with the atmosphere and, hence, the more it penetrates to deeper layers.
Since the galactic cosmic ray spectrum extends to higher energies, it is reasonable that the maximum of the ionization due to galactic cosmic rays is expected to lower atmosphere altitudes with respect to the ones where maximum ionization due to SEP-atmosphere interaction appears. Finally, in the context of planetary space weather, we find that for conditions similar to those during the SEP event in May 2012, the maximum ionization in the Venusian atmosphere, appearing at the altitude of $\sim 78 \mathrm{~km}$, is by a factor of up to $\sim 100$ above the expected ionization due to the galactic cosmic ray background. For conditions similar to those during the SEP event in October 1989, the enhancement in the maximum ionization rate, appearing at the altitude of $\sim 94 \mathrm{~km}$, is almost 5 orders of magnitude higher than the average background rate due to the galactic cosmic rays.

\section{Considerations in the context of planetary space weather in view of future observations}

The fluxes and energy spectra of SEPs vary greatly with time and may produce ionization at low altitudes in a planetary atmosphere (Leblanc et al., 2002; Brain et al., 2009; Nordheim et al., 2015). For example, in the single event simulated by Leblanc et al. (2002), the ionization rate in the Martian atmosphere was greatest at $90-100 \mathrm{~km}$, similarly to the findings by Brain et al. (2009) corresponding to a different SEP event. For Venus, the results obtained with DYASTIMA for both SEP cases studied in the current paper, show a clear dependence of the ionization processes on the space conditions around the planet and in particular on the considered SEP energy spectrum. Our simulations indicate that the harder the SEP spectrum is assumed to be, the lower the altitude of the maximum ionization is. Equivalently to the circumterrestrial space weather, therefore, planetary space weather on Venus could be the driver for numerous processes related to atmospheric cascades and energy deposition. At the altitude of maximum ionization induced by galactic cosmic rays ( 63-64 km), a surplus in the ionization is expected in the case of an SEP event. In particular, for conditions similar to the ones during the SEP on 17 May 2012, at the altitude of maximum ionization due to galactic cosmic rays, an ionization surplus ranging between 82 and $112 \%$ is expected. Similarly, for conditions similar to the ones during the SEP in October 1989, an ionization surplus ranging between 236 and $319 \%$ is expected at this altitude. Although these speculations do not have a strong statistical accuracy, given the limited number of the studied SEP event cases, they provide evidence of a strong influence of the space weather conditions upon the Venus environment, sensed even at relatively low altitudes in the atmosphere.

Low-altitude $(<\sim 80-100 \mathrm{~km})$ atmospheric ionization measurements on Venus are currently not existent (we note that the available ionospheric radio sounding observations performed with the radio science experiment VeRa on Venus 
Express (Peter et al., 2014) covered the region with altitudes $>\sim 100 \mathrm{~km})$. Therefore, a comparison between the results presented in the current paper and real measurements is currently not possible. Nevertheless, there is some indirect observational evidence that SEP precipitation can affect the lower ionosphere of Mars. In particular, the radar sounder MARSIS finds some strong signal attenuation after SEP events (Withers, 2011). Moreover, the radio science experiment MaRS has found some disturbances in the lower ionosphere, and one of the possible explanations was that of SEP precipitation (Peter et al., 2016).

Future in situ ion detection, radar sounder observations of ionospheric attenuation, as well as radio occultation measurements during SEP events could provide information on the ionization resulting from the precipitation of these energetic particles in the Venusian atmosphere. In this context, knowledge of the electron density profiles is important for drawing conclusions about the lowest frequency for which the degradation of radio communication by ionospheric attenuation during SEP events is minor (Withers, 2011). The results of the ion production rates presented in the current paper could serve as a first-order feedback in this direction. For example, based on our estimated maximum ionization rate during an SEP event, we can calculate the expected peak electron density. In particular, if we assume that all ions are rapidly converted into $\mathrm{O}_{2}^{+}$ions (Schunk and Nagy, 2009), consistent with model results of the lower Venusian ionosphere showing an $\mathrm{O}_{2}^{+}$/electron ratio of $>90 \%$ at the ionospheric main peak (Fox and Sung, 2001), then the expected peak electron density at solar zenith may be given by the following formula (Peter et al., 2014):

$n_{e}=\sqrt{\frac{P_{t}}{a_{\mathrm{O}_{2}^{+}}}}$,

where $P_{t}$ is the maximum value of the total ion-electron production rate (in $\mathrm{cm}^{-3} \mathrm{~s}^{-1}$ ) and $a_{\mathrm{O}_{2}^{+}}$is the $\mathrm{O}_{2}^{+}$dissociative recombination coefficient. (Note that the large percentage of $\mathrm{O}_{2}^{+}$in the Venusian ionosphere around the main peak allows us to assume, at first approximation, that every ion produced instantly becomes an $\mathrm{O}_{2}^{+}$ion and that the dissociative recombination of $\mathrm{O}_{2}^{+}, \mathrm{O}_{2}^{+}+e^{-} \rightarrow \mathrm{O}+\mathrm{O}$, is the only loss process for electrons). Considering the estimated maximum ion-electron production rates for both SEP events studied in the current paper (see Sect. 3) and assuming that $a_{\mathrm{O}_{2}^{+}}=2 \times 10^{-7} \mathrm{~cm}^{3} \mathrm{~s}^{-1}$ (Withers, 2011), we estimate a peak electron density equal to $4 \times 10^{4} \mathrm{~cm}^{-3}$ (for conditions similar to those during the SEP in 2012) and equal to $2 \times 10^{5} \mathrm{~cm}^{-3}$ (for conditions similar to those during the SEP in 1989). We note, however, that since it is unlikely that all energy is consumed for the ionization of the Venusian atmosphere, the peak electron densities calculated here are probably overestimations. Further simulations, possibly including (a) ionospheric chemistry that may lead to electron densities not proportional to the energy deposition rate and/or (b) secondary electron production by electron impact (Wedlund et al., 2011), are necessary in order to draw firm conclusions for the electron density profile in the Venusian atmosphere. It is worth noting, however, that the SEP flux is in general highly variable (Mason et al., 1999); hence, the results in the current study should be considered as a first-order identification and quantification of changes in the Venusian atmosphere during two representative SEP events. In the future, theoretical space weather investigations on Venus can provide feedback in two directions: (a) to uncover the details of the variations of the plasma-atmosphere interactions and their impact on the gas composition and spatial redistribution; (b) to provide constraints in the means of ionized particle radiation during different periods of solar activity.

As in the Earth's case (Verronen et al., 2002), SEP events may also affect the concentrations of middle atmospheric constituents on Venus. Although in the current study we do not examine this aspect of the interaction, our results provide a feedback that may be of help during the development of future sophisticated ion and neutral models. In particular, the ion production rate at different atmospheric layers estimated in this study is a quantity that is strongly related to the deposition rate of energy at each altitude and, hence, with the further ionization and dissociative ionization processes of individual constituents. Whereas the effects of galactic cosmic rays and SEPs can be very strong locally in the polar cap regions of the Earth, affecting the physical-chemical and electrical properties of the ionosphere and atmosphere at these regions (Velinov et al., 2013), on Venus their actual impact is expected to expand over the whole dayside atmosphere. Therefore, during the planning of future missions to Venus and the determination of observation strategies, the expected space weather conditions and their variability on different timescales have to be taken into account.

We underline that the results obtained with DYASTIMA correspond to average conditions on the low-latitude Venusian dayside atmosphere; nevertheless, our code allows the model application for any set of galactic cosmic ray and/or solar plasma conditions as well as atmosphere composition.

\section{Conclusions}

The estimations presented in this paper show that any variability in the energy spectrum and integrated flux of the SEPs arriving in the vicinity of Venus is likely to result in variations of the cascade properties and particle ionization. Aplin (2013) had previously suggested that such ionization processes may have effects on the local neutral densities, cloud formation and chemistry in deep atmospheric layers. It is therefore of significant importance to understand and quantify such planetary space weather phenomena on Venus in the context of future mission (e.g. the Deep Atmosphere Venus Investigation of Noble gases, Chemistry, and Imaging 
(DAVINCI); the Venus Emissivity, Radio Science, InSAR, Topography, and Spectroscopy mission (VERITAS)) preparation and also data interpretations of previous missions (e.g. Venus Express).

Through the application of the DYASTIMA code, the SEP-induced ionization profiles in the Venusian atmosphere were estimated and approximate constraints in the expected surpluses were provided. Our main conclusions can be summarized as follows:

1. During SEP events, a surplus in the ionization rates in the Venusian atmosphere is expected; such a surplus is highly variable, depending strongly on the SEP energy spectra. Based on the examined SEP cases, the ionization rate during SEP events is expected to be higher than the background ionization due to galactic cosmic rays, by a factor ranging between $10^{2}$ and $\sim 10^{5}$, during the event's main phase.

2. For both SEP events under study, the maximum ionization appears at altitudes which are by $\sim 6-30 \mathrm{~km}$ higher than the one where the maximum ionization due to galactic cosmic rays appears (i.e. $63-64 \mathrm{~km}$ ).

3. At the altitude of $\sim 63-64 \mathrm{~km}$, the presence of an SEP event can induce a surplus in the ionization rate of up to $319 \%$ with respect to its background due to the galactic cosmic-ray-atmosphere interactions.

Such speculations can be useful in the context of future planetary space weather studies (Lilensten et al., 2014; Plainaki et al., 2016) and preparation for future missions to Venus. Cosmic rays may have a significant role in the ionic and electronic charges for charge separation arising from dynamical processes in the Venus cloud altitudes and likely in the generation of Venus lightning (Borucki et al., 1982; Levin et al., 1983; Yair, 2012; Yair et al., 2008; Nordheim et al., 2015; Michael et al., 2009). For example, Levin et al. (1983), assuming that charge separation and lightning discharges occur on other planets in ways similar to those in which they occur on Earth, concluded that lightning on Venus will probably be found in clouds that are located in regions of convection such as those observed downwind of the subsolar point. Moreover, Michael et al. (2009) carried out modelling of cloud particle charging in the Venusian atmosphere, discussing implications for lightning. The estimated presence of finite charges in the Venusian atmosphere, due to the gas interaction with SEPs, makes the charging of Venus clouds due to solar particle attenuation and degradation possible. Although further studies are necessary in order to investigate this phenomenon, the results presented in the current study may be useful feedback in this direction.
Acknowledgements. C. Plainaki thanks F. González-Galindo (IAACSIC, Spain) and R. Orosei (INAF - Istituto di Radioastronomia, Italy) for useful discussions on ionosphere measurements and M. Storini (INAF-IAPS, Italy) for interesting discussions on atmosphere ionization by galactic cosmic rays. P. Paschalis thanks J. Apostolakis and the Geant4 team for providing basic routines used in the DYASTIMA application.

The topical editor, E. Roussos, thanks the two anonymous referees for help in evaluating this paper.

\section{References}

Agostinelli, S., Allison, J., Amako, K., Apostolakis, J., Araujo, H., Arce, P., Asai, M., Axen, D., Banerjee, S., Barrand, G., Behner, F., Bellagamba, L., Boudreau, J., Broglia, L., Brunengo, A., Burkhardt, H., Chauvie, S., Chuma, J., Chytracek, R., Cooperman, G., Cosmo, G., Degtyarenko, P., Dell'Acqua, A., Depaola, G., Dietrich, D., Enami, R., Feliciello, A., Ferguson, C., Fesefeldt, H., Folger, G., Foppiano, F., Forti, A., Garelli, S., Giani, S., Giannitrapani, R., Gibin, D., Gómez Cadenas, J. J., González, I., Gracia Abril, G., Greeniaus, G., Greiner, W., Grichine, V., Grossheim, A., Guatelli, S., Gumplinger, P., Hamatsu, R., Hashimoto, K., Hasui, H., Heikkinen, A., Howard, A., Ivanchenko, V., Johnson, A., Jones, F. W., Kallenbach, J., Kanaya, N., Kawabata, M., Kawabata, Y., Kawaguti, M., Kelner, S., Kent, P., Kimura, A., Kodama, T., Kokoulin, R., Kossov, M., Kurashige, H., Lamanna, E., Lampén, T., Lara, V., Lefebure, V., Lei, F., Liendl, M., Lockman, W., Longo, F., Magni, S., Maire, M., Medernach, E., Minamimoto, K., Mora de Freitas, P., Morita, Y., Murakami, K., Nagamatu, M., Nartallo, R., Nieminen, P., Nishimura, T., Ohtsubo, K., Okamura, M., O’Neale, S., Oohata, Y., Paech, K., Perl, J., Pfeiffer, A., Pia, M. G., Ranjard, F., Rybin, A., Sadilov, S., Di Salvo, E., Santin, G., Sasaki, T., Savvas, N., Sawada, Y., Scherer, S., Sei, S., Sirotenko, V., Smith, D., Starkov, N., Stoecker, H., Sulkimo, J., Takahata, M., Tanaka, S., Tcherniaev, E., Safai Tehrani, E., Tropeano, M., Truscott, P., Uno, H., Urban, L., Urban, P., Verderi, M., Walkden, A., Wander, W., Weber, H., Wellisch, J. P., Wenaus, T., Williams, D. C., Wright, D., Yamada, T., Yoshida, H., Zschiesche, D., and G EANT4 Collaboration: GEANT4: a simulation toolkit, Nuclear Instruments and Methods in Physics Research A, 506, 250-303, doi:10.1016/S0168-9002(03)01368-8, 2003.

Allison, J., Amako, K., Apostolakis, J., Araujo, H., Arce Dubois, P., Asai, M., Barrand, G., Capra, R., Chauvie, S., Chytracek, R., Cirrone, G. A. P., Cooperman, G., Cosmo, G., Cuttone, G., Daquino, G. G., Donszelmann, M., Dressel, M., Folger, G., Foppiano, F., Generowicz, J., Grichine, V., Guatelli, S., Gumplinger, P., Heikkinen, A., Hrivnacova, I., Howard, A., Incerti, S., Ivanchenko, V., Johnson, T., Jones, F., Koi, T., Kokoulin, R., Kossov, M., Kurashige, H., Lara, V., Larsson, S., Lei, F., Link, O., Longo, F., Maire, M., Mantero, A., Mascialino, B., McLaren, I., Mendez Lorenzo, P., Minamimoto, K., Murakami, K., Nieminen, P., Pandola, L., Parlati, S., Peralta, L., Perl, J., Pfeiffer, A., Pia, M. G., Ribon, A., Rodrigues, P., Russo, G., Sadilov, S., Santin, G., Sasaki, T., Smith, D., Starkov, N., Tanaka, S., Tcherniaev, E., Tome, B., Trindade, A., Truscott, P., Urban, L., Verderi, M., Walkden, A., Wellisch, J. P., Williams, D. C., Wright, D., 
and Yoshida, H.: Geant4 developments and applications, IEEE T. Nucl. Sci., 53, 270-278, doi:10.1109/TNS.2006.869826, 2006.

Aplin, K. L.: Atmospheric Electrification in the Solar System, Surv. Geophys., 27, 63-108, doi:10.1007/s10712-005-0642-9, 2006.

Aplin, K. L.: Electrifying Atmospheres: Charging, Ionisation and Lightning in the Solar System and Beyond, Springer Briefs in Astronomy, Springer Dordrecht Heidelberg New York London, 13-20, ISBN-13: 978-94-007-6633-4, 2013.

Battistoni, G., Ferrari, A., Muraro, S., and Sala, P. R.: Atmospheric muon simulation using the FLUKA MC Model, Nuclear Physics B Proceedings Supplements, 168, 286-288, doi:10.1016/j.nuclphysbps.2007.02.025, 2007.

Borucki, W. J.: Comparison of Venusian lightning observations, Icarus, 52, 354-364, doi:10.1016/0019-1035(82)90118-X, 1982.

Borucki, W., Levin, Z., Whitten, R., Keesee, R.: Predicted electrical conductivity between 0 and $80 \mathrm{~km}$ in the venusian atmosphere, Icarus 321, 302-321, 1982.

Brain, D. A., Delory, G. T., Lillis, R. J., Luhmann, J. G., Bougher, S. W. and Parkinson, C. D.: Energy deposition by energetic protons in the upper atmosphere of Mars, Eos Trans. AGU, 90, Fall Meet. Suppl., Abstract P11B-1215, 14-18 December 2009.

Cramp, J. L., Duldig, M. L., Flückiger, E. O., Humble, J. E., Shea, M. A., and Smart, D. F.: The October 22, 1989, solar cosmic ray enhancement: An analysis of the anisotropy and spectral characteristics, J. Geophys. Res., 102, 24237-24248, doi:10.1029/97JA01947, 1997.

Dartnell, L. R., Nordheim, T. A., Patel, M. R., Mason, J. P., Coates, A. J., and Jones, G. H.: Constraints on a potential aerial biosphere on Venus: I. Cosmic rays, Icarus, 257, 396-405, 2015.

Desorgher, L., Flückiger, E. O., Gurtner, M., Moser, M. R., and Bütikofer, R.: Atmocosmics: a Geant 4 Code for Computing the Interaction of Cosmic Rays with the Earth's Atmosphere, International Journal of Modern Physics A, 20, 6802-6804, doi:10.1142/S0217751X05030132, 2005.

Dorman, L. I.: Cosmic Rays in the Earth's Atmosphere and Underground, Kluwer Academic Publishers, Dordreht/London/New York, ISBN-13: 978-1-4020-2113-8, 2004.

Dorman, L., Paschalis, P., Plainaki, C., and Mavromichalaki, H. Estimation of the cosmic ray ionization in the Earth's atmosphere during GLE71, 34th ICRC, 30 July-6 August 2015, PoS(ICRC2015) 202, available at: http://pos.sissa.it/cgi-bin/ reader/conf.cgi?confid=236, 2015.

Dubach, J., Whitten, R. C., and Sims, J. S.: The lower ionosphere of Venus, Planet. Sci., 22, 525-536, doi:10.1016/00320633(74)90087-7, 1974.

Duplissy, J., Merikanto, J., Franchin, A., Tsagkogeorgas, G., Kangasluoma, J., Wimmer, D., Vuollekoski, H., Schobesberger, S., Lehtipalo, K., Flagan, R. C., Brus, D., Donahue, N.M., Vehkamäki, H., Almeida, J., Amorim, A., Barmet, P., Bianchi, F., Breitenlechner, M., Dunne, E. M., Guida, R., Henschel, H., Junninen, H., Kirkby, J., Kürten, A., Kupc, A., Määttänen, A., Makhmutov, V., Mathot, S., Nieminen, T., Onnela, A., Praplan, A.P., Riccobono, F., Rondo, L., Steiner, G., Tome, A., Walther, H., Baltensperger, U., Carslaw, K. S., Dommen, J., Hansel, A., Petäjä, T., Sipilä, M., Stratmann, F., Vrtala, A., Wagner, P. E., Worsnop, D. R., Curtius, J., and Kulmala, M.: Effect of ions on sulfuric acid-water binary particle formation II: Experimental data and comparison with QC-normalized classi- cal nucleation theory, J. Geophys. Res. Atmos., 121, 1752-1775, doi:10.1002/2015JD023539, 2016.

Ehresmann, B., Burmeister, S., Wimmer-Schweingruber, R. F., and Reitz, G.: Influence of higher atmospheric pressure on the Martian radiation environment: Implications for possible habitability in the Noachian epoch, J. Geophys. Res., 116, A10106, doi:10.1029/2011JA016616, 2011.

Fesefeldt, H. C.: GHEISHA program, Technical Report PITHA 8502, III Physikalisches Institut, RWTH Aachen Physikzentrum, 5100 Aachen, Germany, September 1985.

Fox, J. L. and Sung, K. Y.: Solar activity variations of the Venus thermosphere/ionosphere, J. Geophys. Res., 106, 21305-21335, 2001.

Fujii, Z. and McDonald, F. B.: Radial intensity gradients of galactic cosmic rays (1972-1995) in the heliosphere, J. Geophys. Res., 102, 24201-24208, doi:10.1029/97JA01871, 1997.

Gilli, G., López-Valverde, M. A., Peralta, J., Bougher, S., Brecht, A., Drossart, P., and Piccioni, G.: Carbon monoxide and temperature in the upper atmosphere of Venus from VIRTIS/Venus Express non-LTE limb measurements, Icarus, 248, 478-498, doi:10.1016/j.icarus.2014.10.047, 2015.

Gopalswamy, N., Xie, H., Akiyama, S., Yashiro, S., Usoskin, I. G., and Davila, J. M.: The first ground level enhancement event of solar cycle 24: direct observation of shock formation and particle release heights, Astrophys. J. Lett., 765, L30, doi:10.1088/20418205/765/2/L30, 2013.

Gronoff, G., Lilensten, J., Desorgher, L., and Flückiger, E.: Ionization processes in the atmosphere of Titan. I. Ionization in the whole atmosphere, Astron. Astrophys., 506, 955-964, doi:10.1051/0004-6361/200912371, 2009a.

Gronoff, G., Lilensten, J., and Modolo, R.: Ionization processes in the atmosphere of Titan. II. Electron precipitation along magnetic field lines, Astron. Astrophys., 506, 965-970, doi:10.1051/00046361/200912125, 2009b.

Gronoff, G., Mertens, C., Lilensten, J., Desorgher, L., E. Flückiger, and Velinov, P.: Ionization processes in the atmosphere of Titan (Research Note). III. Ionization by high-Z nuclei cosmic rays, Astron. Astrophys., 529, A143, doi:10.1051/00046361/201015675, 2011.

Gronoff, G., Norman, R. B., and Mertens, C. J.: Computation of cosmic ray ionization and dose at Mars. I: A comparison of HZETRN and Planetocosmics for proton and alpha particles, Adv. Space Res., 55, 1799-1805, doi:10.1016/j.asr.2015.01.028, 2015.

Gurnett, D. A., Zarka, P., Manning, R., Kurth, W. S., Hospodarsky, G. B., Averkamp, T. F., Kaiser, M. L., and Farrell, W. M.: Nondetection at Venus of high-frequency radio signals characteristic of terrestrial lightning, Nature, 409, 313-315, 2001.

Gurtner, M., Desorgher, L., Flückiger, E. O., and Moser, M. R.: Simulation of the interaction of space radiation with the Martian atmosphere and surface, Adv. Space Res., 36, 2176-2181, doi:10.1016/j.asr.2005.05.120, 2005.

Gurtner, M., Desorgher, L., Flückiger, E.O., Moser, M.R.: A Geant4 application to simulate the interaction of space radiation with the Mercurian environment, Adv. Space Res., 37, 1759-1763, 2006.

Heck, D., Knapp, J., Capdevielle, J. N., Schatz, G., and Thouw, T.: CORSIKA: a Monte Carlo code to simulate extensive air showers. Forschungszentrum Karlsruhe GmbH, Karlsruhe (Germany), 
Feb 1998, V + 90 p., TIB Hannover, D-30167 Hannover (Germany), 1998.

Hudson, H. S.: Solar physics: Solar flares add up, Nature Physics, 6, 637-638, doi:10.1038/nphys1764, 2010.

Keating, G. M., Bertaux, J. L., Bougher, S. W., Dickinson, R. E., Cravens, T. E., and Hedin, A. E.: Models of Venus neutral upper atmosphere - Structure and composition, Adv. Space Res., 5, 117-171, doi:10.1016/0273-1177(85)90200-5, 1985.

Kliore, A. J., Moroz, V. I., and Keating, G. M.: The Venus International Reference Atmosphere, Adv. Space Res., 5, 1-305, 1985.

Ksanfomaliti, L. V., Vasilchikov, N. M., Ganpantserova, O. F., Petrova, E. V., Suvorov, A. P., Filippov, G. F., Iablonskaia, O. V., and Iabrova, L. V.: Electrical discharges in the atmosphere of Venus, Soviet Astronomy Letters, 5, 122-126, 1979.

Leblanc, F., Luhmann, J. G., Johnson, R. E., and Chassefiere, E.: Some expected impacts of a solar energetic particle event at Mars, J. Geophys. Res., 107, 1058, doi:10.1029/2001JA900178, 2002.

Levin, Z., Borucki, W. J., and Toon, O. B.: Lightning generation in planetary atmospheres, Icarus, 56, 80-115, 1983.

Li, C., Firoz, K. A., Sun, L. P., and Miroshnichenko, L. I.: Electron and proton acceleration during the first ground level enhancement event of solar cycle 24, Astrophys. Journal, 770, 34, doi:10.1088/0004-637X/770/1/34, 2013.

Lilensten, J., Coates, A. J., Dehant, V., Dudok de Wit, T., Horne, R. B., Leblanc, F., Luhmann, J., Woodfield, E., and Barthélemy, M.: What characterizes planetary space weather?, Astron. and Astrop. Rev., 22, 79, doi:10.1007/s00159-014-0079-6, 2014.

Luhmann, J. G., Ledvina, S. A., and Russell, C. T.: Induced magnetospheres, Adv. Space Res., 33, 1905-1912, doi:10.1016/j.asr.2003.03.031, 2004.

Määttänen, A., Guilbon, S., Stolzenbach, A., Bekki, S., and Montmessin, F.: VenLA: The LATMOS Venus cloud model, EPSC2015-634, 2015.

Mahieux, A., Vandaele, A. C., Robert, S., Wilquet, V., Drummond, R., López Valverde, M. A., López Puertas, M., Funke, B., and Bertaux, J. L.: Rotational temperatures of Venus upper atmosphere as measured by SOIR on board Venus Express, Planet. Sci., 113, 347-358, doi:10.1016/j.pss.2014.12.020, 2015.

Mason, G. L., Cohen, C. M. S., Cumming, C., Dwyer, J. R., Gold, R. E., Krimigis, S. M., Leske, R. A., Mazur, J. E., Mewald, R. A., Mobius, E., Popeck, M., Stone, E. C., von Rosenving, T. T., and Wiedenbeck, M. E.: Particle acceleration and sources in the November 1997 solar energetic particle event, Geophys. Res. Let., 26, 141-144, 1999.

Mavromichalaki, H., Papaioannou, A., Plainaki, C., Sarlanis, C., Souvatzoglou, G., Gerontidou, M., Papailiou, M., Eroshenko, E., Belov, A., Yanke, V., Flückiger, E. O., Bütikofer, R., Parisi, M., Storini, M., Klein, K.-L., Fuller, N., Steigies, C. T., Rother, O. M., Heber, B., Wimmer-Schweingruber, R. F., Kudela, K., Strharsky, I., Langer, R., Usoskin, I., Ibragimov, A., Chilingaryan, A., Hovsepyan, G., Reymers, A., Yeghikyan, A., Kryakunova, O., Dryn, E., Nikolayevskiy, N., Dorman, L., and Pustil'Nik, L.: Applications and usage of the real-time Neutron Monitor Database, Adv. Space Res., 47, 2210-2222, doi:10.1016/j.asr.2010.02.019, 2011.

Merikanto, J., Duplissy, J., Määttänen, A., Henschel, H., Donahue, N. M., Brus, D., Schobesberger, S., Kulmala, M., and Vehkamäki, H: Effect of ions on sulfuric acid-water binary par- ticle formation I: Theory for kinetic and nucleation-type particle formation and atmospheric implications, J. Geophys. Res. Atmos., 121, 1736-1751, doi:10.1002/2015JD023538, 2016.

Michael, M., Tripathi, S. N., Borucki, W. J., and Whitten, R. C.: Highly charged cloud particles in the atmosphere of Venus, J. Geophys. Res., 114, E04008, doi:10.1029/2008JE003258, 2009.

Miroshnichenko, L. I., De Koning, C. A., and Perez-Enriquez, R.: Large solar event of September 29, 1989: ten years after, Space Sci. Rev., 91, 615-715, 2000.

Mishev, A. L., Kocharov, L. G., and Usoskin, I. G.: Analysis of the ground level enhancement on 17 May 2012 using data from the global neutron monitor network, J. Geophys. Res.-Space, 119, 670-679, doi:10.1002/2013JA019253, 2014.

Nordheim, T. A., Dartnell, L. R., Desorgher, L., Coates, A. J., and Jones, G. H.: Ionization of the venusian atmosphere from solar and galactic cosmic rays, Icarus, 245, 80-86, doi:10.1016/j.icarus.2014.09.032, 2015.

Nymmik, R.: Initial conditions for radiation analysis: Models of galactic cosmic rays and solar particle events, Adv. Space Res., 38, 1182-1190, doi:10.1016/j.asr.2006.07.002, 2006.

Odstrcil, D.: Modeling 3-D solar wind structure, Adv. Space Res., 32, 497-506, doi:10.1016/S0273-1177(03)00332-6, 2003.

Paschalis, P., Mavromichalaki, H., Dorman, L. I., Plainaki, C., and Tsirigkas, D.: Geant4 software application for the simulation of cosmic ray showers in the Earth's atmosphere, New Astronomy, 33, 26-37, doi:10.1016/j.newast.2014.04.009, 2014.

Peralta, J., López-Valverde, M. A., Gilli, G., and Piccialli, A.: Dayside temperatures in the Venus upper atmosphere from Venus Express/VIRTIS nadir measurements at $4.3 \mu \mathrm{m}$, Astron. Astrophys., 585, A53, doi:10.1051/0004-6361/201527191, 2016.

Peter, K., Pätzold, M., Molina-Cuberos, G., Witasse, O., GonzaìlezGalindo, F., Withers, P., Bird, M. K., Häusler, B., Hinson, D. P., Tellmann, S., and Tyler, G. L.: The dayside ionospheres of Mars and Venus: Comparing a one-dimensional photochemical model with MaRS (Mars Express) and VeRa (Venus Express) observations, Icarus, 233, 66-82, 2014.

Peter, K., Pätzold, M., Molina-Cuberos, G., Witasse, O., Tellmann, S., Häusler, B., and Bird, M.: The origin of small scale disturbances in the lower ionosphere of Mars, EGU General Assembly 2016, Geophysical Research Abstracts, 18, EGU2016-1040, Vienna, Austria, 17-22 April 2016.

Piccialli, A., Montmessin, F., Belyaev, D., Mahieux, A., Fedorova, A., Marcq, E., Bertaux, J.-L., Tellmann, S., Vandaele, A. C., and Korablev, O.: Thermal structure of Venus nightside upper atmosphere measured by stellar occultations with SPICAV/Venus Express, Planet. Sci., 113, 321-335, doi:10.1016/j.pss.2014.12.009, 2015.

Plainaki, C., Belov, A., Eroshenko, E., Mavromichalaki, H., and Yanke, V.: Modeling ground level enhancements: Event of 20 January 2005, J. Geophys. Res.-Space, 112, A04102, doi:10.1029/2006JA011926, 2007.

Plainaki, C., Mavromichalaki, H., Belov, A., Eroshenko, E., and Yanke, V.: Modeling the solar cosmic ray event of 13 December 2006 using ground level neutron monitor data, Adv. Space Res., 43, 474-479, doi:10.1016/j.asr.2008.07.011, 2009.

Plainaki, C., Mavromichalaki, H., Belov, A., Eroshenko, E., Andriopoulou, M., and Yanke, V.: A New Version of the Neutron Monitor Based Anisotropic GLE Model: Application to GLE60, Solar Phys., 264, 239-254, doi:10.1007/s11207-010-9576-6, 2010. 
Plainaki, C., Mavromichalaki, H., Laurenza, M., Gerontidou, M., Kanellakopoulos, A., and Storini, M.: The Ground-level Enhancement of 2012 May 17: Derivation of Solar Proton Event Properties through the Application of the NMBANGLE PPOLA Model, Astrophys. Journal, 785, 160, doi:10.1088/0004637X/785/2/160, 2014.

Plainaki, C., Laurenza, M., Mavromichalaki, H., Storini, M., Gerontidou, M., Kanellakopoulos, A., Andriopoulou, M., Belov, A., Eroshenko, E., and Yanke, V.: Derivation of relativistic SEP properties through neutron monitor data modeling, J. Phys. Conf. Ser., 632, 012076, doi:10.1088/1742-6596/632/1/012076, 2015.

Plainaki, C., Lilensten, J., Radioti, A., Andriopoulou, M., Milillo, A., Nordheim, T. A., Dandouras, I., Coustenis, A., Grassi, D., Mangano, V., Massetti, S., Orsini, S., and Lucchetti, A.: Planetary Space Weather: Scientific Aspects and Future Perspectives, Journal of Space Weather and Space Climate, doi:10.1051/swsc/2016024, in press, 2016.

Russell, C. T., Luhmann, J. G., and Strangeway, R. J.: The solar wind interaction with Venus through the eyes of the Pioneer Venus Orbiter, Planet. Sci., 54, 1482-1495, doi:10.1016/j.pss.2006.04.025, 2006.

Russell, C. T., Zhang, T. L., Delva, M., Magnes, W., Strangeway, R. J., Wei, H. Y.: Lightning on Venus inferred from whistler-mode waves in the ionosphere, Nature, 450, 661-662, doi:10.1038/nature05930, 2007.

Schunk, R. W. and Nagy, A. F.: Ionospheres: Physics, Plasma Physics, and Chemistry, second ed. Cambridge Univ. Press, Cambridge and others, ISBN-13: 978-0-521-87706-0, 2009.

Seiff, A., Schofield, J. T., Kliore, A. J., Taylor, F. W., and Limaye, S. S.: Models of the structure of the atmosphere of Venus from the surface to 100 kilometers altitude, Adv. Space Res., 5, 3-58, doi:10.1016/0273-1177(85)90197-8, 1985.

Taylor, F. W., Diner, D. J., Elson, L. S., Hanner, M. S., McCleese, D. J., Martonchik, J. V., Reichley, P. E., Houghton, J. T., Bradley, S. E., Delderfield, J., Schofield, J. T., and Ingersoll, A. P.: Infrared remote sounding of the middle atmosphere of Venus from the Pioneer orbiter, Science, 203, 779-781, doi:10.1126/science.203.4382.779, 1979a.

Taylor, F. W., Diner, D. J., Elson, L. S., McCleese, D. J., Martonchik, J. V., Delderfield, J., Bradley, S. P., Schofield, J. T., Gille, J. C., and Coffey, M. T.: Temperature, cloud structure, and dynamics of Venus middle atmosphere by infrared remote sensing from Pioneer Orbiter, Science, 205, 65-67, doi:10.1126/science.205.4401.65, 1979 b.

Tylka, A. J., Dietrich, W. F., Boberg, P. R., Smith, E. C., and Adams, J. H.: Single event upsets caused by solar energetic heavy ions, IEEE T. Nucl. Sci., 43, 2758-2766, doi:10.1109/23.556863, 1996.

Tylka, A. J., Adams, J. H., Boberg, P. R., Brownstein, B., Dietrich, W. F., Flueckiger, E. O., Petersen, E. L., Shea, M. A., Smart, D. F., and Smith, E. C.: CREME96: a revision of the Cosmic Ray Effects on Micro-Electronics code, IEEE T. Nucl. Sci., 44, 2150 2160, doi:10.1109/23.659030, 1997.
Upadhyay, H. O. and Singh, R. N.: Cosmic ray ionization of lower Venus atmosphere, Adv. Space Res., 15, 99-108, doi:10.1016/0273-1177(94)00070-H, 1995.

Usoskin, I. G., Gladysheva, O. G., and Kovaltsov, G. A.: Cosmic ray-induced ionization in the atmosphere: spatial and temporal changes, J. Atmos. Sol.-Terr. Phy., 66, 1791-1796, doi:10.1016/j.jastp.2004.07.037, 2004.

Usoskin, I. G., Kovaltsov, G. A., and Mironova, I. A.: Cosmic ray induced ionization model CRAC:CRII: An extension to the upper atmo- sphere, J. Geophys. Res.-Atmos., 115, D10302, doi:10.1029/2009JD013142, 2010.

Velinov, P. I. Y., Asenovski, S., Kudela, K., Lastovicka, J., Mateev, L., Mishev, A., and Tonev, P.: Impact of cosmic rays and solar energetic particles on the Earth's ionosphere and atmosphere, Journal of Space Weather and Space Climate, 3, A14, doi:10.1051/swsc/2013036, 2013.

Verronen, P. T., Turunen, E., Ulich, Th., and Kyrölä, E.: Modelling the effects of the October 1989 solar proton event on mesospheric odd nitrogen using a detailed ion and neutral chemistry model, Ann. Geophys., 20, 1967-1976, doi:10.5194/angeo-201967-2002, 2002.

Wedlund, S. C., Gronoff, G., Lilensten, J., Ménager, H., and Barthélemy, M.: Comprehensive calculation of the energy per ion pair or $\mathrm{W}$ values for five major planetary upper atmospheres, Ann. Geophys., 29, 187-195, doi:10.5194/angeo-29-187-2011, 2011.

Withers, P.: Attenuation of radio signals by the ionosphere of Mars: Theoretical development and application to MARSIS observations, Radio Sci., 46, RS2004, doi:10.1029/2010RS004450, 2011.

Yair, Y.: New results on planetary lightning, Adv. Space Res., 50, 293-310, doi:10.1016/j.asr.2012.04.013, 2012.

Yair, Y., Fischer, G., Simões, F., Renno, N., and Zark, P.: Updated Review of Planetary Atmospheric Electricity, Space Sci. Rev., 137, 29-49, doi:10.1007/s11214-008-9349-9, 2008.

Zhang, T. L., Delva, M., Baumjohann, W., Auster, H.-U., Carr, C., Russell, C. T., Barabash, S., Balikhin, M., Kudela, K., Berghofer, G., Biernat, H. K., Lammer, H., Lichtenegger, H., Magnes, W., Nakamura, R., Schwingenschuh, K., Volwerk, M., Vörös, Z., Zambelli, W., Fornacon, K.-H., Glassmeier, K.-H., Richter, I., Balogh, A., Schwarzl, H., Pope, S. A., Shi, J. K., Wang, C., Motschmann, U., and Lebreton, J.-P.: Little or no solar wind enters Venus' atmosphere at solar minimum, Nature, 450, 654-656, doi:10.1038/nature06026, 2007. 